\title{
¿TENDRÁ LA «CONVERGENCIA DE TECNOLOGÍAS» (CT) Y LA «MEJORA TÉCNICA DEL SER HUMANO» UN IMPACTO SIMILAR AL DARWINISMO? (IMPLICACIONES Y CONSIDERACIONES FILOSÓFICAS)*
}

\section{WILL «CONVERGING TECHNOLOGIES» AND «HUMAN ENHANCEMENT» HAVE A SIMILAR IMPACT AS DARWINISM? (SOCIAL IMPLICATIONS AND PHILOSOPHICAL CONSIDERATIONS)}

\author{
Nicanor URSUA** \\ Departamento de Filosofía \\ Universidad del País Vasco/Euskal Herriko Unibertsitatea
}

\begin{abstract}
RESUMEN: Se expone el concepto de "convergencia de tecnologías» (converging technologies) a partir de investigaciones llevadas a cabo en Estados Unidos de América y de Europa y se asocia este concepto con la idea de la «mejora del ser humano» (el tema del «human enhancement» («technische Verbesserung des Menschen»), es decir, del aumento tecnológico o «mejora» de las capacidades humanas y la modificación de la corporalidad y del intelecto humano). Se ofrece una reflexión filosófico-social naturalista sobre esta temática que para muchos autores supone un impacto en la
\end{abstract}

* Este trabajo se inscribe en el marco del Proyecto de Investigación EHU07/34, financiado por la Universidad del País Vasco/Euskal Herriko Unibertsitatea.

** e-mail: nicanor.ursua@ehu.es 
sociedad académica similar al darwinismo en el siglo XIX al aparecer en el debate de la CT el concepto de transhumanismo o tecnofuturismo que podría conducir a la transformación de la especie humana.

Palabras Clave: Convergencia de tecnologías, tecnologías de mejora del ser humano, capacidades cognitivas humanas, naturalismo, justicia distributiva, tecnofuturismo, transhumanismo,

ABSTRACT: The concept of "converging technologies» is being used since the investigations in USA and Europe associated this concept (converging technologies) to the idea of "human enhancement»; in other words, the improvement of human capacities, corporal and also intellectual modification. This article presents a social and philosophical naturalistic approach on the subject which has been considered, among the academic society, to have the same influence as Darwin's theories in the 19th century; particularly on the debate about converging technologies and the concept of transhumanism or tecnofuturism which could lead to the transformation of the human species.

KEYWORDS: Converging technologies, improving human performance, human cognitive capacities, naturalism, distributive justice, transhumanism, technofuturism.

\section{Introducción}

¿Qué se quiere decir cuando se habla de "convergencia de tecnologías" (Converging Technologies» (CT))? El concepto de "convergencia de tecnologías» está asociado normalmente a la ciencia y a la tecnología y un ejemplo de ello es el uso que se hace de este concepto en los equipamientos informáticos y en la electrónica de uso doméstico. Aquí, se usa este concepto para describir la interacción de diferentes disciplinas científico-tecnológicas para abordar e intentar solucionar problemas comunes a todas ellas mediante la cooperación trans e interdisciplinar. Podríamos decir que el concepto de convergencia se usa aquí para describir el desarrollo de distintas tecnologías que se centran en una combinación de evidencias de investigación desde las diferentes disciplinas entre sistemas vivos 
y sistemas artificiales para el diseño de nuevos dispositivos que permitan expandir o mejorar las capacidades cognitivas y comunicativas, la salud y las capacidades físicas de las personas y generar de este modo un mayor bienestar social.

En el centro de este nuevo concepto están las relaciones interactivas, las sinergias o fusiones de amplios campos de investigación y desarrollo, tales como: la nanociencia y la nanotecnología, la biotecnología y las ciencias de la vida, las tecnologías de la información y de la comunicación, las ciencias cognitivas y las neurotecnologías, la robótica y la inteligencia artificial. Se ha caracterizado el debate sobre la convergencia de tecnologías como «el foro para explorar el futuro impacto de toda la ciencia y de la ingeniería».

Se señala como punto de partida del debate sobre la CT la iniciativa sobre política de investigación y de tecnología de los Estados Unidos de América del Norte del año 2001. (Ver al respecto: Roco, Mihail C./Bainbridge, William S., 2003. Estos autores fueron los primeros en utilizar el concepto de CT. Este concepto se ha expresado gráficamente como el «NBIC tetrahedron»). Estamos hablando, en concreto, de la iniciativa "NBIC initiative» (iniciativa "nano, bio, info, cogno») y, sobre todo, del aspecto que interesa aquí que es el de la «improving human performance» $y$ del tema de la "human enhancement» («technische Verbesserung des Menschen»), es decir, del aumento tecnológico o «mejora» de las capacidades humanas y la modificación de la corporalidad y del intelecto humano. (Ver: Fuchs, Michael; Lanzerath, Dirk; Hillebrand, Ingo; Runkel, Thomas; Balcerak, Magdalena; Schmitz, Barbara, 2002; Aguiló, Jordi, 2005; Andler, Daniel; Barthelmé, Simon; Beckert, Bernd; Blümel, Clemens; Coenen, Christopher; Fleischer, Torsten; Friedewald, Michael; Quendt, Christiane; Rader, Michael; Simakova, Elena; Woolgar Steve, 2008; Fleischer, Torsten; Quendt, Christiane; Rader, Michael, 2008; Grunwald, Armin, 2007a; 2007b; 2007c; 2008). Se volverá a tratar el tema del «enhancement» un poco más adelante.

La Comisión Europea en el marco del programa «Citizens and Governance in a Knowledge based Society» (VI Programa Marco) ha solicitado que se estudien los posibles efectos de las nuevas tecnologías convergentes sobre la «sociedad europea del conocimiento». El documento de la Comisión Europea del año 2003, publicado en 2004, por el «High Level Expert Group (HLEG)» (Comisión de Grupo de Expertos convocados y financiados por la Unidad K2: «Society and Technology Foresight» de la Dirección K: «Knowledge-based Economy and 
Society» de la Dirección de Investigación de la Comunidad Europea): «Foresighting the New Technology Wave»: «Converging Technologies — Shaping the Future of European Societies (Nano-Bio-Info-Cogno-Socio-Antropo-Phil)», editado por Nordmann, Alfred (CTEKS, http://ec.europa.eu/research/conferences/ 2004/ntw/pdf/final_report_en.pdf 30.12. 2008) (ver también: Coenen, Christopher; Rader, Michael; Fleischer, Torsten, 2004, que exponen la posición de este grupo europeo), a diferencia de la iniciativa americana que centra sus esfuerzos en el «enhancement» (mejora) del ser humano individual y defiende una «nueva unidad de la ciencia», caracterizada por el reduccionismo radical, llevando todo a la nanoescala (defiende que la base común de todas las ciencias está a escala nano; el documento americano se podría caracterizar por las siguientes palabras: «Si el científico cognitivo puede concebirlo, el nano puede construirlo, el bio puede aplicarlo y el info puede monitorizarlo y construirlo»), adopta un enfoque en el que la CT responde a las necesidades y demandas de la sociedad y está caracterizado por un marco de interdisciplinaridad y multidiscipilinaridad en el que la Filosofía también tiene una tarea muy específica al ayudar a precisar y explicar el proceso de convergencia científico-tecnológico, al analizar los nuevos modos de producción del conocimiento, al examinar las «culturas epistémicas» de las diferentes disciplinas participantes (Knorr-Cetina, Karin, 1999), obtener visiones de las nuevas tecnologías y sus impactos a nivel social y ético y contribuir a clarificar la «nueva» autocomprensión del ser humano.

La Unión Europea, una vez secuenciado el genoma humano y tras los avances en áreas como la neurología y la ciencia cognitiva, creyó que había llegado el momento de desarrollar, dentro del VI Programa Marco, un proyecto europeo sobre la mente humana, el «Human Mind Project» o HMP, para desarrollar una línea de investigación interdisciplinaria sobre lo que significa ser humano, publicado en 2005 por un Grupo de Expertos de alto nivel (Report of a NEST (New and Emerging Science and Technology) High-Level Expert Group: HLEG): What it means to be human. Origins and Evolution for Human Higher Cognitive Faculties.(ftp://ftp.cordis.europa.eu/pub/nest/docs/whatitmeanstobehuman_ b5_eur21795_en.pdf). (1. 12. 2008). La investigación se ha de centrar, según este informe, que de acuerdo al grupo de expertos afectará a la forma en que vivimos y podría tener en el siglo XXI un impacto comparable al que tuvo el darwinismo en el siglo XIX, en cinco amplias áreas temáticas, a saber: 1) la genética de la cognición humana, a saber, cómo nuestra especie, a pesar de su similitud genética con otros simios, ha evolucionado y mantiene una extraordinaria men- 
te ciertamente compleja, 2) la evolución de la mente, a saber, cómo diferentes experiencias vitales influyen en el desarrollo, maduración y envejecimiento del cerebro humano normal, 3) el proceso del pensamiento, a saber, cómo los nuevos «insights» en el razonamiento, aprendizaje y memoria impactan e impactarán en la educación, la comunicación y el desarrollo de tecnologías inteligentes, 4) la motivación y la toma de decisiones, a saber, estudio de los «insights» que motivan a las personas a cooperar o a actuar de manera contraria y qué factores nos influyen a tomar las decisiones que tomamos y 5) el contexto cultural, a saber, cómo la mente humana se manifiesta como cultura, cómo las culturas cambian y cómo perduran; qué conductas y maneras de pensar son parte de nuestra cultura y cuáles son parte de la naturaleza. Desde el punto de vista de la CT, se han de tener en cuenta la genética, la neurobiología, las ciencias cognitivas, las ciencias de la conducta animal y humana, la paleoantropología, la historia, los diferentes modelos y la filosofía de la mente. Lo interesante del informe es la propuesta de colaboración entre especialistas de las ciencias naturales y de las ciencias humanas y sociales y la insistencia de que quiénes somos y cómo pensamos y ciertamente cómo nos comportamos está fundamentalmente unido a nuestra historia evolutiva compartida y naturaleza humana (que no se ha de olvidar como hacen algunas filosofías) y a nuestro entorno físico, cultural y social. Si conocemos nuestra mente adecuadamente, sólo entonces estaremos en condiciones de poder orientarnos en este mundo y realizar cambios pertinentes. No se ha de descuidar, pues, lo natural y lo cultural.

En este contexto hemos de ubicar el proyecto «Tecnologías Convergentes NBIC» (2005) y, sobre todo, "CONTECS - Converging Technologies and their impact on Social Sciences and Humanities» (2008). Estos proyectos, que nos sirven como una de las bases para la reflexión, y en concreto el último, es un «Specific Support Action», que dentro del VI Programa Marco de Investigación, ha sido financiado por la Comisión Europea de Febrero de 2006 a Abril de 2008 y ha sido realizado por el Institut für Technikfolgenabschätzung und Systemanalyse en el Forschungszentrum Karlsruhe (ITAS), el Fraunhofer-Institut für System und Innovationsforschung (FhG-ISI Karlsruhe), como coordinador del proyecto, la Saïd Business School (Oxford) y la Ecole Normale Supérieure (París). (Ver: Andler, Daniel; Barthelmé, Simon; Beckert, Bernd; Blümel, Clemens; Coenen, Christopher; Fleischer, Torsten; Friedewald, Michael; Quendt, Christiane; Rader, Michael; Simakova, Elena; Woolgar Steve, 2008; Fleischer, Torsten; Quendt, Christiane; Rader, Michael, 2008). 


\section{2. ¿Por qué es interesante profundizar y reflexionar sobre los documentos reseñados sobre $\mathrm{CT}$ en el año del bicentenario de Ch. Darwin?}

La atenta lectura y reflexión del documento de la «National Science Foundation» (Roco, Mihail/Bainbridge, William, 2003) y de CTEKS, así como del proyecto CONTECS, entre otras muchas publicaciones que reseñamos aquí, nos llevan a pensar en promesas y situaciones de desarrollo futuristas para la humanidad, que contienen temas muy relevantes social, ética, ontológica y antropológicamente en la medida que la CT puede abrir nuevos campos para la elección y la acción humana (función emancipatoria al ofrecérsenos nuevas oportunidades para la acción: "mejora técnica», remodelación del cuerpo y de la mente, lo que puede conducir a pensar que estamos ante una "culturización» de los elementos hasta ahora naturales del ser humano: el «argumento naturalista» de que los seres humanos son fisiológicamente lo que la evolución ha hecho de ellos, y así debe de ser, ya no nos sirve por más tiempo).

A su vez, la CT y sus expectativas futuristas pueden «disolver» los valores, las certezas y las autocomprensiones tradicionalmente mantenidas, en tanto en cuanto aumenta «la contingencia de la conditio humana», lo que puede requerir una nueva orientación en esta nueva situación contingencial que amplía las posibles elecciones entre varias opciones y con ello una disminución de la dependencia de la naturaleza y de las tradiciones de la humanidad, pero que puede ir acompañado de situaciones ambiguas o inciertas para el ser humano al cuestionar certezas tradicionalmente admitidas. (Grunwald, Armin, 2007a, 3-7; 2007b, 381383; 2007c, 949-953).

¿Esteremos determinados científico-tecnológicamente o existirán espacios de libertad basados en aspectos sociales y éticos? ¿Se solventarán todos los problemas humanos científico-tecnológicamente? ¿No estaremos asistiendo pasivamente a una tecnificación del ser humano ("cyborgs», simbiosis ser humano-máquina)? ¿Estamos asistiendo al proceso transhumano o posthumano (movimientos que argumentan a favor de la «mejora humana» de manera radical, algo que podría conducir a la transformación y superación de la especie humana mediante una civilización totalmente técnica)? ¿Qué quiere decir aquí «human enhancement» («mejora humana»)? 


\section{CT y la «mejora» de las capacidades humanas}

La «mejora del ser humano» («human enhancement»; «technische Verbesserung des Menschen»), tanto física como intelectual, se puede afirmar que no es para nada un tema nuevo, es más, es un tema muy viejo, tan viejo como la existencia del ser humano. (Nuestros antepasados bíblicos Adán y Eva ya quisieron superarse a sí mismos intentando convertirse en dioses). ¿Cuántas personas están satisfechas con su cuerpo y con su mente? ¿No nos gustaría ser diferentes? ¿No nos gustaría ser omniscientes y omnipotentes? ¿No nos gustaría vencer a la edad y hasta la muerte? Parece que se está dando hoy más que nunca, lo que ya se ha denominado como un cierto «descontento normativo» con una o varias características específicas corporales independientemente de una psicopatología. (Fuchs, Michael, et al., 2002, 73).

Nuestra insatisfacción corporal es superada o corregida, a nivel individual, mediante la cirugía plástica y cosmética (cirugía corporal), que cada vez cobra más auge en nuestra sociedad a nivel individual y que tiene cada vez más una alta repercusión económica y que, por otra parte, no está mal vista o del todo no mal vista. Hay que señalar, sin embargo, que el concepto de apariencia externa es un concepto subjetivo y que se da, en general, en la comparación con los demás. En este campo, se ha de diferenciar entre aquellas personas a las que les puede ayudar una pequeña corrección quirúrgica y aquellas que se someten a una operación con dismorfofóbias que necesitan más un tratamiento psiquiátrico. Desde un punto de vista ético-social, se ha de analizar si el paciente se somete a la operación voluntariamente y autónomamente, o si está obedeciendo a normas sociales externas muy discutibles (tiranía de las normas sociales y cultura de consumo). A veces, existe la ilusión de que se puede controlar la vida y la vejez y que se puede mejorar de manera contínua.Una imagen y apariencia externa joven gana muchos enteros en nuestra sociedad, que olvida frecuentemente el aspecto interno del ser humano. Para no caer en complicidades, es necesario analizar el modelo interactivo entre paciente y médico que puede ser paternalista, informativo, interpretativo y deliberativo. Este último introduce la consideración de los valores del paciente y del médico con relación a la salud y al bienestar humano. Como este tipo de cirugía está, por lo general, fuera de la teleología médica tradicional, se puede argumentar que la cirugía cosmética no pertenece, en líneas generales, a la necesidad médica, pues no tiene como meta la salud, sino la belleza. La finalidad médica se orienta en realidades físicas objetivamente com- 
probables, mientras que la cirugía cosmética se orienta más en el cumplimiento de deseos ciertamente subjetivos. (Ver: Fuchs, Michael, et al. 2002, 71-85).

Nuestros límites físico-naturales los intentamos «mejorar», por lo general, mediante el entrenamiento constante. En el deporte, por ejemplo, existe el entrenamiento intensivo o el uso de "medios técnicos» para mejorar el rendimiento. El lema del deporte ha sido siempre: «citius, altius, fortius»: más rápido, más alto, más fuerte. El Comité Olímpico Internacional define el dopaje como: 1) «el uso de una sustancia o método que es potencialmente peligrosa para la salud del atleta o es capaz de mejorar su rendimiento y 2) la presencia en el cuerpo del atleta de una sustancia prohibida o evidencia de su uso o evidencia del uso de un método prohibido». En algunos casos el uso del dopaje (estimulantes, narcóticos, anabolizantes, diuréticos, péptido hormonas, hormonas de crecimiento, dopaje génico, etc.) está considerado muy poco deportivo y está penalizado. La discusión sobre el dopaje se encuadra dentro del «juego limpio» ("fair-play»), de la igualdad de oportunidades (es decir, las mismas condiciones externas) y de la autenticidad. (Ver: Fuchs, Michael, et al. 2002, 85-106). Estos investigadores en el año 2002 tematizaron el campo de acción del «enhancement» (mejora) además de en la cirugía plástica y cosmética y del deporte, vistos más arriba, en el campo de la mejora técnico-génica, en el tratamiento de las hormonas de crecimiento en pediatría y en la mejora de la psíque mediante psicofármacos.

A nivel colectivo, la humanidad, que ha deplorado sus defectos en términos morales o civilizatorios, los ha intentado corregir o «mejorar», sobre todo desde la Ilustración, mediante la educación personal y la cultura con la esperanza de mejorar la condición humana y de la sociedad en general.

Hoy, se tiene la esperanza, sin embargo, de que «mejoraremos» las capacidades humanas, y por ende, las acciones sociales, no desde la educación y la cultura, sino mediante el desarrollo y la aplicación convergente de las nonotecnologías, las biotecnologías, las tecnologías genéticas, la aplicación de las tecnologías de la información y de la comunicación, las ciencias cognitivas, las neurotecnologías y las investigaciones sobre el cerebro humano.

Si nos atenemos a lo afirmado por Roco, Mihail/Bainbridge, William, 2003, «la mejora técnica» de las capacidades humanas, se centra en capacidades técnicas cuantitativas que van desde la ampliación de las facultades sensoriales huma- 
nas (por ejemplo, la mejora del ojo, del oído humano, etc.) pasando por la memoria y el cerebro (implantación de chips, neuroimplantes, etc.) y llegando hasta el retardo o la desaparición del envejecimiento humano, lo que aumenta ciertamente la "contingencia de la conditio humana». (Grunwald, Armin, 2007a, 4-5; 2007b, 382-383; 2007c, 950-951).

El proyecto CONTECS, 2008, 10, con ayuda de expertos de diferentes campos científico-tecnológicos, ha identificado 8 áreas de $\mathrm{I}+\mathrm{D}$ en las que es posible ubicar casi toda la investigación y desarrollo en el campo de la CT, a saber: 1) las neurociencias y la «mejora» del cerebro; 2) la «mejora» física y biomedicina; 3) la biología sintética; 4) el interfaz ser humano-máquina; 5) los diferentes sensores; 6) los modelos de reconocimiento; 7) los modelos basados en la computerización del mundo y 8) los robots y el software inteligente y artefactos.

\section{El papel del «human enhancement technologies» («tecnologías de mejora humana») y algunas consideraciones ético-sociales}

Una de las preguntas fundamentales con relación al «human enhancenment» («mejora humana») es saber cuáles son las metas y objetivos de esta mejora humana. Esta pregunta está, a su vez, relacionada con visiones sociales y políticas, con factores ideológicos, con conceptos antropológicos y con el mantenimiento de valores fundamentales que configuran los debates y las actividades científico-tecnológicas que, sin duda, pueden influir en la definición de conceptos tales como salud, normalidad, terapia, perfectibilidad, etc.

Las «tecnologías de mejora humana» van desde proyectos concretos como la alteración del metabolismo de los soldados o el desarrollo de sofisticados interfaces cerebro-máquina hasta el desarrollo de imágenes de un «futuro posthumano» en el que una civilización terrestre simbiótica ser humano-máquina se expande fuera de nuestro espacio. Algunos visionarios posthumanistas y tecnofuturistas consideran incluso como posibilidad la sustitución total de la humanidad por máquinas inteligentes y otros afirman que la hibridación del ser humano-máquina es la única opción que le queda a la humanidad, más que la «mejora», para evitar un escenario futuro cercano que podría marginar al ser humano. Este transhumanismo cree en una civilización radicalmente nueva técnica que va más allá 
de la humanidad actual. (CONTECS, 2008, 35. Sobre el tecnofuturismo posthumanístico, ver: Coenen, Christopher, 2006).

Un grupo de expertos reunidos en Bruselas en septiembre de 2008 (Coenen, Christopher, 2008) para analizar este tema, afirman que hay que distinguir en «human enhancement» entre la mejora de las especies, con sus tintes eugenésicos, algo nada interesante como visión guía por razones históricas, pragmáticas y metafísicas, y la mejora de los individuos. (Sobre la discusión del tema de la genética humana si va hacia una nueva eugenesia desde abajo, ver: Irrgang, Bernhard, 2002; Romeo-Casabona, Carlos, 2004, 325, este último afirma que las intervenciones genéticas en la línea somática o germinal o en el proceso de la selección biológica son de carácter duradero e incluso irreversible y se podrían considerar eugenésicas si están unidas directamente a la reproducción humana. Ver también J. Habermas, 2009).

Se comentó en ese encuentro que una visión guía más apropiada a nivel europeo era considerar la mejora tanto a nivel del bienestar individual como de la cohesión social, examinando las relaciones entre los factores sociales e individuales. Coenen Christopher (2008a) prefiere hablar de «optimización humana» más que de «mejora humana».

Algunos expertos afirmaron que el discurso sobre la «mejora humana» está fuertemente influenciado por una «fe acrítica en la ciencia» y que las visiones alternativas de futuro y propuestas para resolver los problemas de la sociedad era algo que estaba totalmente ausente o se descuidaba en ese discurso. Muchas personas toman este tema con mucha ilusión y esperanza, pues escuchan únicamente lo que dicen los agentes políticos o los miembros de la élite tecnocrática y, por ello, es necesario imaginarse alternativas y obtener visiones sociales relacionadas con la ciencia y la tecnología con mayor participación pública. Se necesita, por tanto, una visión y orientación que guíe los desarrollos ulteriores de investigación y las tecnologías que son relevantes en el contexto de la «mejora humana» y esa visión y orientación debería estar fundamentada, sin duda, en la perspectiva social enfocada a la cohesión social y a la justicia distributiva como marco de la elección individual.

Aunque todavía existe una laguna entre las visiones existentes sobre CT y sus aplicaciones reales (las aplicaciones futuras de la CT pueden ser todavía muy 
difíciles de predecir), y la configuración concreta y la capacidad de resultados de las técnicas no son del todo conocidas, se puede afirmar, según los estudios de Andler, Daniel; Barthelmé, Simon; Beckert, Bernd; Blümel, Clemens; Coenen, Christopher; Fleischer, Torsten; Friedewald, Michael; Quendt, Christiane; Rader, Michael; Simakova, Elena; Woolgar Steve, 2008, 22-26; Fleischer, Torsten; Quendt, Christiane; Rader, Michael, 2008, 76-77, que una gran parte de los discursos éticos y de la evaluación de tecnologías (Technology Assessment) se concentran en la pregunta general sobre el desarrollo de las técnicas, y en este caso en concreto, en las oportunidades y en los riesgos (caracterización del riesgo, evaluación del riesgo, gestión del riesgo, comunicación del riesgo) de las «técnicas de mejora humana». También será necesario tener en cuenta, en este tema, el principio de precaución, tal como lo ha definido la Unión Europea.

Grunwald, Armin, 2007a, 7-13; 2007b, 383-391; 2007c, 953-955, propone el estudio del papel de la comunicación sobre el futuro (comunicación futurista) para proveer una nueva orientación en vista de la creciente contingencia de la condición humana en la que ya no existe un estado ideal de la constitución física o mental del ser humano y en la que el estado ideal normal se puede optimizar. Esta comunicación contribuye: 1) a aumentar la contingencia (función catalizadora o medial), 2) señala la creciente contingencia (función indicadora) y 3) contribuye a gestionar las consecuencias (función orientadora). Esta orientación no se obtiene automáticamente, pues puede darse una gran ambivalencia (expectativas de salvación y nuevos paraísos, así como posibles miedos y/o catástrofes). Para hacer un uso constructivo del potencial de orientación en la comunicación futurista es necesario proponer un nuevo instrumento como es la evaluación de la visión futurista. (Ver también Coenen, Christopher, 2004, donde propone la evaluación de la visión para examinar el nanofuturismo como parte del tecnofuturismo).

Esta evaluación puede constituir un nuevo elemento dentro de la caja de herramientas de la evaluación de tecnologías, señalada más arriba, en la que pueden y deben contribuir la reflexión filosófica, los estudios de filosofía de la tecnología y de la ciencia, así como las ciencias empíricas y de la comunicación, al analizar las visiones como un medio de comunicación con sus contenidos y consecuencias cognitivas y evaluativas para sí contribuir a hacer posible una discusión transparente y racional. 
Los pasos a dar en la evaluación de la visión futurista serían: 1) análisis epistemológico de la visión, 2) evaluación de la visión para categorizar y juzgar los aspectos cognitivos en base a su grado de realización y 3) gestión de la visión para decidir y actuar de manera racional.

Aunque no siempre es fácil separar la ética como campo de investigación de los temas sociales y legales (sobre las perspectivas legales en los nuevos tratamientos psiquiátricos y en la investigación relacionada con esos tratamientos, así como la «mejora de las capacidades psíquicas» y la necesidad de establecer nuevas decisiones legales, ver: Romeo-Casabona, Carlos, 2004), y en este caso, de las reflexiones filosóficas y antropológicas, existen una serie de preguntas relacionadas con el «human enhancement» («mejora del ser humano») en estas expectativas (o incluso posibilidades) visionarias que se podrían exponer de la manera siguiente y que esperan, por supuesto, una respuesta:

¿Qué significa hoy, en el bicentenario de Ch. Darwin, ser humano? ¿Dónde reside la dignidad humana? ¿Qué es hoy la naturaleza, y en concreto, la naturaleza humana? ¿Se puede y se debe poner en peligro o incluso eliminar la «naturalidad» del ser humano mediante la mejora técnica? ¿Qué es la identidad personal? ¿Qué quiere decir autodeterminación y libre albedrio? ¿Qué sentimientos de "autoengaño» tienen las personas acerca de la mejora cognitiva en la ética del trabajo, en la aspiración personal, en el esfuerzo y en la autenticidad? ¿Qué hacer con la coerción o presión (aspectos sociales y económicos, tales como la desventaja por falta de «mejora», implicaciones económicas, etc.)? ¿Cómo distinguir la imagen natural de la imagen no natural, las modificaciones permanentes de las temporales? ¿Qué ocurre con el tema de la «justicia distributiva», a saber, quién puede tener acceso a la «mejora»? ¿Se dará, por tanto, una división en la sociedad entre los que pueden y no pueden tener acceso a la mejora? ¿Qué consecuencias e implicaciones tiene para nuestro concepto de humanidad y para la sociedad del futuro? ¿Existen o deben de existir límites a la «mejora técnica» del ser humano (a esa "tecnificación del ser humano»)? ¿Bajo qué criterios se ha de realizar? ¿Cómo se ha de decidir sobre los criterios? ¿Hasta dónde pueden y deben ir los seres humanos en su reconstrucción del cuerpo y de la mente? ¿Existen riesgos en la "mejora técnica»? ¿Qué actitud tomamos ante las «mejoras técnicas»? ¿Bastará con el «consentimiento informado»? ¿Se puede hacer algo normativamente? ¿Cómo se han de tratar los conflictos con las normas, las creencias y los valores? ¿Habrá que tener en cuenta también otras culturas? ¿Habrá que impli- 
car a todos los agentes sociales en el debate? ¿Se ha de destinar financiación pública en esas «mejoras técnicas»? ¿Dónde reside la responsabilidad personal en el diseño de nuestro cuerpo y mente? ¿Puede la filosofía aportar una orientación en todas estas preguntas para enfocar adecuadamente estos temas? (Ver también al respecto, Grunwald, Armin, 2007a, 5-6; 2007b, 383; 2007c, 950, 952; 2008).

\section{EI naturalismo y el modelo determinista del ser humano}

«Naturalizar» un campo o dominio es afirmar, en síntesis, que pertenece a la naturaleza y que, por lo tanto, puede ser abordado y explicado mediante la ayuda de las denominadas ciencias naturales, sin recurrir a nada extramundano. En este sentido, la CT enfatiza el carácter del naturalismo y considera que los seres humanos, incluidas sus estructuras cognitivas, son entidades de la naturaleza, sometidas a sus fuerzas, e interactúan constantemente con otras entidades. Las investigaciones científico-naturales y tecnológicas explicarán y «mejorarán» al ser humano, y para ello, la CT cree tener las herramientas adecuadas, tanto a nivel individual como social.

El debate moderno sobre el naturalismo representa la controversia acerca de la interpretación de los resultados de investigación científico-naturales y sus posibles consecuencias para el orden social. Ejemplos muy relevantes de lo señalado lo constituyen, entre otros muy conocidos, las investigaciones y resultados del proyecto genoma humano y ciertos resultados de las investigaciones sobre el cerebro humano, y en este caso en concreto, la "mejora técnica del ser humano", que abre, como ya se ha señalado, nuevas posibilidades de elección con relación a la configuración o «reconstrucción» del cuerpo y de la mente humana, aunque se pueda y se deba dar un «consentimiento informado».

El naturalismo filosófico, por su parte, que asume la postura naturalista frente a la filosofía "más autónoma» (resistencia a admitir categorías externas en su tarea filosófica) y que critica el reduccionismo, se puede caracterizar, grosso modo, como una concepción y un programa, que reclama, al menos cuatro elementos esenciales: 1) una imagen cósmica conjunta, a saber, una imagen del mundo; 2) atribuye al ser humano una parte en el cosmos, pero muy modesta; 3) en ello comprende todas las capacidades del ser humano y 4) sobre estos fundamentos reclama y desarrolla una antropología naturalista, una teoría naturalista del cono- 
cimiento, una metodología naturalista de investigación, una ética naturalista y hasta una estética naturalista.

Según esta posición filosófica, nuestro universo es un sistema causal cerrado $\mathrm{y}$, por tanto, todos sus problemas internos y todas las cuestiones epistemológicas se pueden solucionar con los medios que presenta este sistema cósmico. (Ver: Vollmer, Gerhard, 2003, 362; Kanitscheider, Bernulf, 1994). Gayon, Jean, 2003, plantea las raíces biológicas del proyecto naturalista y su extensión a fenómenos culturales, defendiendo la co-evolución, al igual que Kanitscheider, Bernulf, 1991, 374, el binomio biológico-cultural (gen-cultura), donde ambos planos se interpretan como un sistema acoplado. Se afirma la base biológica portadora del intelecto, donde el propio plano autónomo de la cultura participa también en el proceso evolutivo, lo que significa una convergencia de los modelos biológico y cultural.

Este tipo de filosofía naturalizada no está en contra del naturalismo e intenta seguir un monismo metodológico científico-natural, ontológico y epistemológico, aunque esto no convierte a la filosofía, sin más, en una ciencia natural.

La creciente formalización de la filosofía y de las ciencias sociales, en general, tiene un efecto todavía pequeño, pero constante y demostrable. Al querer explicar fenómenos, cada vez más complejos, crece, sin duda, la exigencia de la formalización y la cuantificación a través de los estudios de las ciencias cognitivas empíricas y de las neurociencias, de la biología evolucionista, de la teoría evolucionista del conocimiento, etc. (Ursua, Nicanor, 1993), traspasando esos resultados de las ciencias naturales a la manera de las ciencias humanas, aunque pueden encontrar aquí reticencias por su reduccionismo ontológico o ser percibidas como amenazantes, pensemos, por ejemplo, en programas de investigación de carácter bio-neuro-cogno evolucionistas que proponen al ser humano como una mera criatura biológica sometida al proceso causal evolutivo, fisiológico y mecánico sin dar lugar a otro tipo de determinación y perdiendo todos sus atributos esenciales. En este sentido, se afirma que el ser humano perdería su libertad o autonomía (libre albedrío).

Como la CT es más una tecnología que una ciencia dependiente de un determinismo material o natural que opera con seres humanos e impone, a su vez, un nuevo determinismo muy efectivo, esta postura es, según algunos investigadores de las ciencias humanas, algo no aceptable y hasta aborrecible. 
Una pregunta fundamental relacionada con el tema de la naturalización, se podría formular de la manera siguiente: ¿Cuándo puede ser aceptable la naturalización? Los investigadores del proyecto CONTECS, 2008, 33, afirman como hipótesis digna de estudio la siguiente: La naturalización es aceptable, tanto por parte de una gran mayoría de investigadores de las ciencias humanas y sociales como por parte de la opinión pública, en tanto en cuanto lo que se naturaliza (en el doble sentido: en el sentido estricto del bio-naturalismo y en el sentido lato del método formal-cuantitativo), es una facultad cognitiva «inferior»o aquella parte de la facultad «superior» que consiste en una forma vacía o en un espacio de posibilidades formales. La naturalización no podría ir más allá de la forma y el contenido afectado. Así, lo que una persona dice, cree o pretende en una ocasión específica no es sujeto de naturalización. Si no se traspasa esa línea, entonces el libre albedrío, la responsabilidad, etc., estarían fuera del alcance de las ciencias naturales y fuera también de la intervención tecnológica.

Si esta hipótesis es falsa, entonces habría que investigar las raíces de la resistencia a la naturalización. Si es verdadera, entonces sería interesante estudiar en todo su detalle cómo la intuición se expresa realmente, en particular, cómo las personas (expertas y no expertas) ponen la línea entre forma y contenido y cómo la intuición mantiene contraejemplos. Habría que estudiar el fenómeno de la intuición, por consiguiente, tanto desde el punto de vista no tutorizado como desde el campo de la filosofía, la epistemología social y desde las ciencias cognitivas (psicología del desarrollo, cognición social, antropología cognitiva y evolucionista).

La afirmación de que al naturalizar al ser humano estaría en peligro o se eliminaría su "naturalidad» por medio de la «mejora técnica» no se puede considerar un argumento fuerte, pues la naturalidad o culturalidad del ser humano está, a su vez, como sabemos, unida a diferentes interpretaciones de la condición humana. Se podría afirmar que el argumento de que no deberíamos mejorar técnicamente las facultades adquiridas biológico-evolutivamente, pues se han desarbolado así y están adaptadas evolutivamente, se podría considerar una «falacia naturalista», según Grunwald, Armin, 2007a, 6, pues de ahí no se sigue nada normativo y además limita las capacidades a las propiedades dadas de manera natural y esto reduciría la humanidad a «una pieza de museo» y negaría el aspecto cultural del ser humano al que pertenece el trascenderse a sí mismo, a saber, pensar más allá de lo dado. De este argumento tampoco se sigue sin más que la «mejora técnica» esté permitida o sea un imperativo tecnológico. Lo que sí se 
requiere son criterios y una sólida orientación para poder decidir personalmente de manera responsable y racional. La pregunta debería ser «mejorar» para qué, con qué finalidad, reflexionar y sopesar los efectos y los riesgos y decidir con un transfondo ético y social.

Por último, con relación al concepto de «human enhancement» (mejora humana) (ver: Fuchs, Michael, et al. 2002, 16-17, 24-25, 44-45) habrá que reflexionar extensamente: 1) sobre el papel que cumple la medicina y la acción médica en el restablecimiento y la conservación de la salud, así como sobre el uso y aplicación, en general, del conocimiento científico-tecnológico, 2) sobre la utilización de los escasos recursos médicos y de investigación con relación a la salud y al bienestar humano, 3) sobre el aspecto de la legitimación ético-social de la «mejora del ser humano» a la luz de los aspectos de la justicia distributiva, como ya se ha señalado, entre personas y regiones y de la igualdad de oportunidades, 4) sobre el modelo de sociedad que deseamos y en la que queremos vivir y 5) sobre qué tipo de ser humano deseamos, con qué dignidad y con qué concepto normativo (necesidad de llevar a cabo una autoevaluación del ser humano y del diseño del mismo).

Se impone, por tanto, una «Filosofía evaluativa» dentro de una concepción evaluativa de la naturaleza para entender «lo que significa ser humano» y obtener así una autocomprensión del ser humano adecuada y una orientación social en la agenda de la política de investigación.

No cabe duda de que estamos ante un cambio radical en la manera de abordar al ser humano y de intentar comprenderlo científico-tecnológicamente en toda su extensión. Quiénes somos, cómo pensamos y actuamos está ciertamente unido a nuestra historia biológico-evolutiva y a nuestro entorno físico y sociocultural actual. Sólo si somos capaces de tejer estas costuras de manera conjunta, y no de manera aislada, estaremos en condiciones de adentrarnos en los misterios de nuestra existencia y seremos capaces también de orientarla en base a valores ético-sociales y de ir solventando muchos de los problemas que nos afligen, como pueden ser, por ejemplo, entre otros, la manera de cómo educar a nuestros niños/as, de cómo aprende el cerebro y podemos aprender, de cómo motivar a nuestros ciudadanos/as a adoptar una vida más sana para sí y su entorno, de cómo nos comportamos y por qué de la manera que lo hacemos, de cómo elaborar normas que nos ayuden a cooperar, etc. 
Ciertamente que la tecnología es necesaria y se ha de desarrollar, pero no lo es todo, ya que no nos dará todas las soluciones que necesitamos, hay que aprender, sin duda, y esto es importante filosóficamente, a cambiar el modo de cómo pensamos y nos comportamos.

La CT nos plantea, por último (ver CONTECS, 2008, 40-41) una serie de exigencias de pensamiento que podemos definir brevemente de la siguiente manera: desde el punto de vista de la Filosofía e Historia de la Ciencia, nos compromete a una serie de retos epistemológicos, de nuevos modos de producción del conocimiento, el status ontológico de los desarrollos de la CT y los nuevos paradigmas de la ciencia y de la tecnología. Desde el punto de vista de la Historia de la Ideas, se han de abordar las nuevas y cambiantes concepciones de las interacciones ser humano-máquina, las interrelaciones utópicas y las visiones escatológicas y tecno científicas. Desde el punto de vista de la Ética, (vio-, nano-, negro-, info.-), se ha de abordar el tema de la justicia distributiva, la dignidad humana, el informe consentido y el libre albedrio. Desde el punto de vista de la Antropología, se han de tener en cuenta las relaciones ser humano-artefacto, el trans y el posthumanismo. A las Ciencias Humanas y Sociales la CT, en general, les plantea el tema de la mediación del diálogo público sobre CT, a saber, mediante conferencias ciudadanas, compromiso de arriba hacia abajo y la evaluación participativa de la tecnología.

\section{Bibliografía}

Aguiló, J. (Coordinador), 2005. Tecnologías Convergentes NBIC. Situación y Perspectiva 2005. Barcelona: CSIC.. (http://nbic.org.es; http://nbic.org.es/institute/downloadseu/NBIC-2005.pdf. 30. 12. 2008).

ANDlER, D.; Barthelmé, Simon; Beckert, Bernd; Blümel, Clemens; Coenen, Christopher; Fleischer, Torsten; Friedewald, Michael; Quendt, Christiane; Rader, Michael; Simakova, Elena; Woolgar Steve (2008): Converging Technologies and their Impact on the Social Siences and Humanities (CONTECTS). An Analysis of Critical Issues and Suggestion for the Future Research Agenda. Final Report. May. (http://www.contecs. fraunhofer.de/images/files/contecs_report_complete.pdf 31.12. 2008).

Coenen, Ch., 2004. «Nanofuturismus: Anmerkungen zu seiner Relevanz, Analyse und Bewertung», en Technikfolgenabschätzung - Theorie und Praxis. Nr. 2, 13. Jg., Juni 2004, 78-85. 
Coenen, Ch.; Rader, Michael; Fleischer, Torsten, 2004. «Of Visions, Dreams and Nightmares: The Debate on Converging Technologies», en TechnikfolgenabschätzungTheorie und Praxis. Nr. 3, 13. Jg., Dezember 2004, 118-125.

Coenen, Ch., 2006. «Der posthumanistiche Technofuturismus in den Debatten über Nanotechnologie und Converging Technologies», en Nordmann, Alfred; Schummer, Joachim; Schwarz, Astrid (Ed.): Nanotechnologien im Kontext: Philosophische, ethische und gesellsachaftliche Perspektiven. Akademische Verlagsgesellschaft. Berlin, 195-222.

- 2008. «Expert Meeting «Human Enhancement». Shifting Boundaries, Changing Concepts: The Challenges of Human Enhancement to Social, (Dis-)Ability, Medical and Ethical Frameworks», en Technikfolgenabschätzung - Theorie und Praxis. Nr. 3, 17. Jg., Dezember 2008, 144-145.

- 2008a. «Die Vollstreckung des Prinzips der Technizität - Anmerkungen zu aktuellen Visionen wissenschaftlich-technischer Konvergenzprozesse». Ponencia en el Congreso Topoi der Rationalität. Technizität, Medialität, Kulturalität. Institut für Philosophie der Universität Potsdam. Potsdam 2008.

EUROPEAN UniOn. Report of a NEST (New and Emerging Science and Technology) High-Level Expert Group (2005, VI Programa Marco): What it means to be human. Origins and Evolution for Human Higher Cognitive Faculties. (ftp://ftp.cordis.europa.eu/ pub/nest/docs/whatitmeanstobehuman_b5_eur21795_en.pdf). (1. 12. 2008).

Fleischer, T.; Quendt, Christiane; Rader, Michael, 2008. «Converging Technologies und die Sozial- und Geisteswissenschaften. Ergebnisse und Erfahrungen aus einem EU-Projekt», en Technikfolgenabschätzung - Theorie und Praxis. Nr. 2, 17. Jg., September 2008, 74-77.

FuCHS, M.; Lanzerath, Dirk; Hillebrand, Ingo; Runkel, Thomas; Balcerak, Magdalena; Schmitz, Barbara, 2002. Enhancement. Die ethische Diskussion über biomedizinische Verbesserung des Menschen. Editado por Deutsches Referenzzentrum für Ethik in den Biowissenschaften. DRZE-Sachstandsbericht 1. Bonn.

GAYON, J., 2003. «Naturalisation de la culture, naturalisation de la philosophie: Enjeux et limites», en Buschslinger, Wolfgang/Lütge, Christoph (2003): Kaltblütig. Philosophie von einem rationalen Standpunkt. Festschrift für Gerhard Vollmer zum 60. Geburstag, Stuttgart/Leipzig: S. Hirzel Verlag., 243-275.

GRUNWALD, A., 2007a. "Converging Technologies for human enhancement - a new wave increasing the contingency of the conditio humand», en http://www.itas.fzk.de/ deu/lit/epp/2007/grun07-pre04.pdf (19.12.2008).

- 2007b. "Converging Technologies: Visions, increased contingencies of the conditio humana, and search for orientation», en Futures, 39 (2007), 380-392. 
— 2007c. "Orientierungsbedarf, Zukunftswissen und Naturalismus. Das Beispiel der «technischen Verbesserung» des Menschen», en Deutsche Zeitschrift für Philosophie, 55 (2007), 949-965.

- 2008. Auf dem Weg in eine nanotechnologische Zukunft. Philosophisch-ethische Fragen, Freiburg: Karl Alber.

Habermas, J., 2009. El futuro de la naturaleza humana. ¿Hacia una engenesia liberal? Barcelona: Paidós.

HLEG, 2004. Converging Technologies. Shaping the Future of European Societies. A Report from the High Level Expert Group on «Foresighting the New Technology Wave».Rapporteur Alfred Nordmann. Bruselas. http://ec.europa.eu/research/ conferences/2004/ntw/pdf/final_report_en.pdf, (30.12.2008).

IRRGANG, B., 2002. Humangenetik auf dem Weg in eine neue Eugenik von unter? Europäische Akademie. Graue Reihe. Nr. 31. Wahrlich Druck. Bad Neuenahr-Ahrweiler.

KANiTSCHEIDER, B., 1991. «Biología evolutiva, ética y destino del hombre», en Folia Humanistica. Tomo XXIX, Nr. 322, 355-381.

- 1994. «Naturalismus und wissenchaftliche Weltorientierung», en Logos. Neue Folge. Band 1. Heft 2, 184-199.

Knorr-Cetina, K., 1999. Epistemic Cultures. How the Sciences make Knowledge, Cambridge Mass.: Harvard University Press.

Roco, M. C.; Bainbridge, William S. (eds.), 2003. Converging Technologies for Improving Human Performance: Nanotechnology, Biotechnology, Information Technology and Cognitive Science. Dordrecht, Boston, London: Kluwer Academic Press. NSF/DOCsponsored report, Arlington, VA: National Science Foundation, June, Online: http://www.wtec.org/ConvergingTechnologies/ (19.12. 2008).

Romeo-Casabona, C., 2004. «Legal perspectives in novel psychiatric treatments and related research», en Poiesis \& Praxis, 2, 315-328.

Ursua, N., 1993. Cerebro y conocimiento. Un enfoque evolucionista, Barcelona: Anthropos.

VOlLmer, G., 2003. «¿Cómo es que podemos conocer el mundo? Nuevos argumentos sobre la teoría evolucionista del conocimiento», en Diálogo Filosófico, 57 (2003), 356-377.

Recibido: $10 / 08 / 2009$

Revisado: 5/10/2009 